\title{
Liver transplantation in oxalosis prior to advanced chronic kidney disease
}

\author{
Jon I. Scheinman
}

Received: 13 October 2009 /Revised: 27 January 2010 / Accepted: 26 February 2010 / Published online: 29 July 2010

(C) IPNA 2010

\begin{abstract}
While curative of the disease, combined kidney and liver transplantation (K/LTx) for primary hyperoxaluria type 1 (PH1) continues to carry with it a risk for patient death of $15-25 \%$, which over time may not differ from that of kidney transplantation alone (KTx). In this editorial, survival data are reviewed as well as the limited data available for kidney graft function, which may favor K/LTx in the short term but is more uncertain in the longer term. The window of opportunity that favors preemptive K/LTx is relatively narrow and is likely even narrower for preemptive isolated LTx. Capability and experience in the medical management of such patients, and the opportunities available, as well as likely patient compliance, so far without supporting data, may be the most important determination of the best strategy for management.
\end{abstract}

Keywords Hyperoxaluria · Liver transplantation · Kidney transplantation $\cdot$ Oxalosis $\cdot$ Preemptive transplant

In this issue of Pediatric Nephrology, Galanti et al. [1] report a remarkable and successful case of primary hyperoxaluria $(\mathrm{PH})$, treated with preemptive [glomerular filtration rate (GFR) $>60 \mathrm{ml} / \mathrm{min}$ ] deceased donor (DD) combined kidney and liver transplantation (DD K/LTx) at age 21 months, following diagnosis at 2 months of age. The diagnosis was made expectantly, due to the post-mortem diagnosis of $\mathrm{PH}$ in a sibling dying of renal failure at 8 months of age. The excellent management and outcome in the second child raises the question as to whether such a preemptive approach should be more generally utilized in PH.

J. I. Scheinman $(\square)$

Fairway, KS 66205, USA

e-mail: jischeinman@gmail.com
Primary hyperoxaluria type 1 (PH1) is an inherited metabolic deficiency of the hepatic peroxisomal enzyme, alanine-glyoxalate-aminotransferase (AGT), resulting in increased oxalate generation, the victim of which is the kidney. Large amounts of oxalate in the urine combine with calcium as insoluble $\mathrm{CaOx}$, resulting in nephrolithiasis, nephrocalcinosis, and usually renal failure. Once the kidney fails, oxalate is no longer adequately excreted, and the elevated plasma oxalate combines with calcium everywhere in the body, termed oxalosis, with resultant organ damage and death. While the currently appropriate cure for the disease is removal and replacement of the defective liver, the choice of therapy depends upon the effect of the disease on the patient, the expectation of progression, and therapy available.

The degree of hyperoxaluria is generally related to the different genetic mutations, whereby oxalate synthesis in certain genotypes is generally greater and in the mistargeting genotypes is usually less. In the latter (homozygous Gly170Arg and Phe152Ile mutations), there may be a variable degree of decrease in oxalate synthesis by pyridoxine, i.e. vitamin B6 (B6), thereby making the disease likely more amenable to successful treatment. These issues have been well reviewed elsewhere [2, 3]. A recent analysis of conservative treatment in children [4] and a comparison with other studies in which conservative treatment has been reported [5] suggest that early diagnosis and treatment is the major factor favoring renal stability. Unfortunately, information on oxalate production and calcium oxalate supersaturation, likely the most important determinants of the medical course, has not been available in any study. All therapy is in fact directed toward those factors: liver transplant addresses oxalate production, and conservative therapy addresses the supersaturation of calcium oxalate. 
The problem to be addressed here is whether, how, and when to apply the "cure", liver transplantation, assuming that a center experienced with the disease is available. This "cure" assumes that kidney failure will occur, and will recur, without that treatment. The predominant strategy for management of PH in Europe, for the past two decades, has been to directly pursue combined K/LTx for patients with renal failure $[6,7]$. The underlying medical milieu is one of relatively uncommon LD (living donor) isolated kidney transplantation (KTx), but a cooperative relationship of physicians with specialized $\mathrm{PH}$ transplant centers in Europe. This has coincided with the intrinsic difficulty in effecting successful DD KTx in patients with PH. For patients with $\mathrm{PH}$, the relatively longer wait for DD KTx, compared to LD, results in a massive buildup of oxalate in the body, whose re-solublization after transplantation, added to endogenous production, easily overwhelms and destroys the transplanted kidney.

In the USA, KTx in many centers has often been blessed with a large number of LD for KTx. Beginning in 1973 [8], we made use of this resource to develop a successful strategy for isolated KTx. Our approach, extrapolated from the strategy and accounts of success by Lynwood Smith [9], addressed the issue of early recurrence with aggressive pretransplant dialysis, early transplantation before oxalate stores accumulated, and vigorous post-transplant management until the released oxalate was minimized.

In order to assess the results of different management strategies, either prospectively or retrospectively, data must be uniformly collected. The results of the European PH1 transplant registry [7] reflect the experiences of the contributing consortia. As the patients were referred for liver transplantation, the results of any prior isolated kidney transplantation were naturally very poor, but may not reflect the results of all such efforts, as is clear from the recent report from France of conservative management [4]. Likewise, in the USA, the results reported by the International Registry for Primary Hyperoxaluria, centered at the Mayo Clinic, depend upon the voluntary contributions of participating physicians [5]. The results of United States Renal Data System (USRDS) and United Network for Organ Sharing (UNOS) databases, which uniformly include data on end-stage renal disease (ESRD) and transplantation in the USA, have been used to estimate overall survival and graft survival for patients with $\mathrm{PH}$.

As summarized in Table 1, there is a degree of variability in the PH patient survival data from the different sources and in the methods, but there is an overall pattern that suggests the hazards of the disease and limitations of therapy. Using the USRDS and UNOS databases, we addressed the overall picture in the USA in 2001, reflecting data through 2000 [10]. This search required an additional search of the USRDS hospitalization data, as the diagnoses initially assigned for the USRDS transplant registries were often incorrect for $\mathrm{PH}$, and liver transplantation was not specifically identified.

We identified 219 patients with PH since 1984. Of these, 60 had no transplant (NoTx), 93 had isolated KTx, and 66 had combined K/LTx. Based on the life table analysis, the survival of both KTx and K/LTx patients were clearly better than that of the NoTX patients at $6(54 \%)$ and $12(41 \%)$ years $(p<0.001)$, but there was no demonstrable statistical survival advantage for either transplant approach in $\mathrm{PH}$ patients. Cibrik et al. [11], concentrating upon graft survival, examined the USRDS data up to 1998 and found no patient survival advantage at 8 years. While the patient survival figures for $\mathrm{PH}$ appear discouraging, other patient groups who received KTx have poorer survival results than the overall population: The overall African-American population $(<55$ years of age) has a survival of $70 \%$ at 12 years, and this is also true for patients with sickle cell disease $63 \%$ [12].

Overall results for the liver transplant procedure (DD LTx) range in the USA, based on UNOS data in the USA [13], at 3 or 5 years, adult or pediatric [14], and in Europe, at 5 years [15], from 75 to $84 \%$ survival. It is then clear that both LTx and ESRD carry a considerable risk for life.

Kidney graft survival has also been addressed for $\mathrm{PH}$ patients. We estimated that kidney graft survival appeared to confer an advantage of K/LTx over isolated KTx at 6 years ( 77 vs. $57 \%$ at 6 years; $p \leq 0.02$ ), but a statistical difference could not be shown at 12 years ( 57 vs. $49 \%$ ) [10]. Cibrik et al. [11] found an advantage for deathcensored K/LTx for graft survival at the 8-year follow-up ( 76 vs. $47.9 \% ; p<0.001$ ), although the higher number of their patients, over a shorter time span, suggests that the diagnoses were not similarly scrutinized. While graft survival results for $\mathrm{PH}$ are not as good as for overall KTx in the USA, problems remain with chronic allograft nephropathy. Repeat transplant can eventually be expected in a large proportion of young patients receiving KTx. There is no data available on the causes of patient death or quality of life from the "systematic" databases.

The European consortium provided a life table analysis for K/LTx patients with $\mathrm{PH}[6,7]$. This registry reflects selected patients, but it additionally contains data on the importance of the condition of patients transplanted to survival that are not available in other datasets. The data presented in 2008 from the oxalosis registry based at the Mayo Clinic [16] suggest an overall patient survival of $76 \%$, with mortality more likely in the K/LTx group. Kidney graft survival was estimated (death censored) at $75 /$ $57 / 43 \%$ for $1 / 3 / 5$ years, respectively, for isolated KTx alone and at $100 / 89$ for $1 / 3$ years for K/LTx, with only two patients at risk at 5 years. 


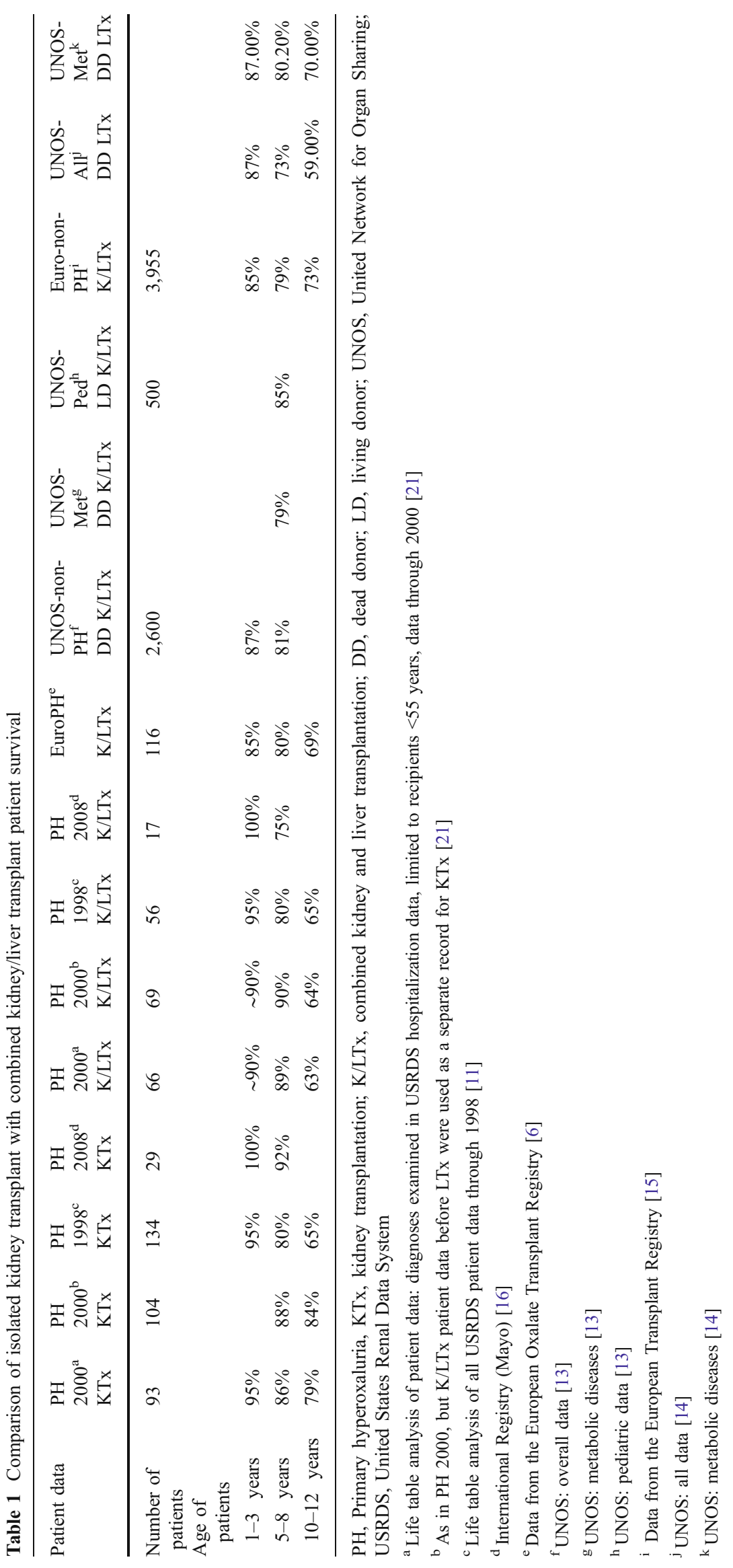


The opportunity to look at specialized centers was provided from a clinic in Hamburg, Germany [17], whose experience with 13 patients was reported. Seven patients underwent K/LTx; all were alive at reporting, with one having required a repeat KTx for "chronic allograft nephropathy". Four patients had isolated, attempted preemptive isolated LTx for PH; one whose GFR was 27 required a later $\mathrm{KTx}$, and the other three were stable with GFR $>80 \mathrm{ml} / \mathrm{min}$. Two patients with isolated LTx, intended to be sequential, died. Excellent results were also reported from San Francisco [18]. The results of the center providing 22 cases to the European PH registry have unfortunately not been separately reported [7].

In summary, of the data currently available (Table 1), it is clear that PH is a known hazard for survival after the development of ESRD. Long-term patient survival after KTx or K/LTx is similar. The excellent recent reviews of $\mathrm{PH}[2,3]$ do not enable a choice of therapy to be made for the individual patient. Quality of life is implied as being better for K/LTx from the "voluntary" reports, but comparable objective data are not available.

Our opinion, and likely a consensus on this issue, is that when the diagnosis of $\mathrm{PH}$ is made after renal failure has developed, it is advisable to determine which factors have resulted in renal failure at that time, i.e. whether it is a precipitating event or an inexorable course suddenly discovered, to estimate the likely body burden of oxalate. The patient who develops ESRD due to oxalate nephropathy would be fortunate to "reset the clock" with the knowledge of how to avoid recurrent kidney failure. This may explain why avoiding any delay in diagnosis was the most important variable in preventing kidney deterioration in the systematic French analysis of conservative treatment in $\mathrm{PH}$ [4].

One potential advantage that can be given to the patient with $\mathrm{PH}$ is knowledge of a response to pyridoxine (vitamin B6). Even a $30 \%$ (partial) response would encourage an attempt at isolated KTx, although there is no controlled data available to support that approach as being distinctively advantageous. The response to B6 can be determined even in ESRD by measuring plasma oxalate before and after the introduction of pyridoxine [19]. There is no theoretical or objective evidence of benefit from pyridoxine, or even from LTx, for other forms of oxalosis, such as $\mathrm{PH} 2$ or enteric hyperoxaluria.

The challenge, apparent since our original report [8], is to prevent recurrent kidney failure. The insoluble oxalate that accumulates in the body after kidney function has fallen to approximately $35 \%$, with the resultant development of plasma supersaturation, resolublizes after plasma levels are lowered by good (transplant) clearance. That oxalate load, added to endogenous production, can be an insurmountable challenge. Therefore, preparation for any kidney transplant requires vigorously addressing that challenge: we arbitrarily required at least 2 weeks of daily prolonged hemodialysis at maximal clearance prior to any planned transplant [20]. In infants or other patients who had been initially begun on peritoneal dialysis, nightly peritoneal dialysis was continued. We suggested that the aggressive dialysis regimen should be instituted almost immediately upon diagnosis in order to avoid the further accumulation of oxalate that occurs during any delay before KTx. There have been attempts to estimate the load of oxalate that is present at the time of transplant, as a determinant of outcome of transplantation, but objective data are unavailable. Our systematic examination of bone marrow (unpublished) associated extensive bone marrow deposition of oxalate with KTx failures. Such an aggressive dialysis strategy would often be impossible with DD KTx, as long delays in obtaining an organ are the norm, and the endurance of dialysis facilities exhausted. Hence, in Europe, with predominant DD Ktx, it is seldom utilized.

The distinctive advantage of adding LTx (with removal of native liver) to KTx is that it is a cure for the increased oxalate production. If the excessive accumulated oxalate can be somehow handled without damage to the kidney, that patient then could embark upon a course expected for a standard KTx recipient. Current cadaveric allocation schemes in the USA allow sufficient advantage to combined K/LTx so that isolated (preemptive) DD KTx is less feasible in PH patients. The absence of a LD then makes LTx almost mandatory. With present immunosuppressive regimens, it is unclear whether simultaneous LTx adds any specific immunological advantage to the slightly higher risks for rejection of DD KTx, or even of LD combined (partial) K/LTx. It is likely, but not conclusive, that graft function is better when LTx is added to KTx.

The major disadvantage of LTx is a likely higher early risk of death. Even in the European PH consortium, overall mortality has been at least $30 \%$, and at least $18 \%$ within the first 2 years. This compares with the findings from overall USRDS and UNOS data for PH in the USA. Using only the more recent time period, 1996-2000, patient survival was statistically equivalent between KTx and K/LTx at 6 years, $86 \%$ [21]. What is not available from the statistical data is the type of strategy and management that was used to develop those results, nor the condition of the patient and oxalate load at the time of transplant.

\section{The choice of management, considered from the standpoint of ethics}

Ethics focuses on beneficence, whereby the choice is the patient's or guardian's, and the physician's counsel must only consider the patient's interests, presenting as objec- 
tively as possible the currently imprecise data. Who then should be initially considered for K/LTx? Most would agree that the patient with ESRD due to oxalate nephropathy due to $\mathrm{PH} 1$, who shows no response to $\mathrm{B} 6$, with no potential living donor for $\mathrm{KTx}$, should receive a DD K/LTx, performed rapidly at a center that has experience with $\mathrm{PH}$, maintaining aggressive dialysis until the time of transplant, and aggressive management afterwards.

In the case of the patient with ESRD who may have at least some response to $\mathrm{B} 6$ and who is receiving aggressive dialysis before transplant and good experience-guided management, LD KTx is a potentially reasonable approach, with the responsibility of informing the donor of the likely greater risk for graft failure. With no B6 response, that approach could still be reasonable, as there are patients who have $\mathrm{PH}$ but who, with careful management, have had no deterioration of native and even transplanted kidney function over many years $[4,5,8]$. The patient must be extremely compliant and willing to trade a likely greater risk of recurrence for a slightly lesser risk of early mortality. The largest renal mass possible should be chosen. In any isolated KTx option, it should be recognized that if the kidney shows deterioration, repeat KTx and the K/LTx approach are still available.

Any K/LTx or isolated KTx require the same careful and aggressive preparation and the same aggressive posttransplant management. The difficulty of distant travel to an experienced transplant center for any DD transplant for $\mathrm{PH}$ requires that the transplant center without such experience should so inform the recipient and seek intimate and continuous guidance.

The use of LD KTx plus partial LD LTx remains problematic, as it creates an added risk to the donor; there will therefore continue to be less likelihood in obtaining such donors, but with fully informed consent, such an approach is reasonable. The use of isolated LTx as a "bridge" to a later KTx has been fraught with problems, as the LTx recipient with continued renal failure and oxalosis is a special challenge [17]. Ongoing routine dialysis will not even prevent the accumulation of normal oxalate synthesis.

The final question, of preemptive isolated LTx as an intentional strategy, before "advanced" chronic kidney disease, presupposes a certainty of progression of the kidney disease as well as the inevitability of recurrence of chronic kidney disease in the transplanted kidney without LTx. Patients with estimated GFR of $>60 \mathrm{ml} / \mathrm{min}$, with a new diagnosis of PH1, might alternately benefit from B6 and other conservative management strategies for a considerable time [4], before taking on the early risk to life of LTx. Current data do not provide a prediction of that time course, which would require some sign of progression of disease, whether by increasing nephrocalcinosis or by decreasing kidney function. Therefore, there is only a narrow window in which isolated LTX may be chosen. Such was done successfully by Galanti et al. [1]. However, a few questions concerning even those excellent results. The excellent course from 2 to 21 months was admirable, but even then, the medical management measures may not have been maximal, nor was there any evidence of progression of kidney disease. Had LTx not been performed at that time, is it certain, and how soon, would renal failure have ensued? Had the child died after the LTx, would the risk of death have been less, 1 or several years later? We have cared for similar PH1 patients with both native kidneys and isolated KTx with similarly excellent courses over the long term. Clearly a solution better than transplant of any sort is preferable, and sought for by many investigators, but thus far has been elusive due to the pharmaceutical industry's reluctance to support them [22] and the slow progress of clinical trials [23].

\section{References}

1. Galanti M, Contreras M (2010) Excellent renal function and reversal of nephrocalcinosis 8 years after isolated liver transplantation in an infant with primary hyperoxaluria type 1. Pediatr Nephrol. doi:10.1007/s00467-010-1593-z

2. Hoppe B, Beck BB, Milliner DS (2009) The primary hyperoxalurias. Kidney Int 75:1264-1271

3. Cochat P, Liutkus A, Fargue S, Basmaison O, Ranchin B, Rolland MO (2006) Primary hyperoxaluria type 1: still challenging! Pediatr Nephrol 21:1075-1081

4. Fargue S, Harambat J, Gagnadoux MF, Tsimaratos M, Janssen F, Llanas B, Bertheleme JP, Boudailliez B, Champion G, Guyot C, Macher MA, Nivet H, Ranchin B, Salomon R, Taque S, Rolland MO, Cochat P (2009) Effect of conservative treatment on the renal outcome of children with primary hyperoxaluria type 1 . Kidney Int 76:767-773

5. Lieske JC, Monico CG, Holmes WS, Bergstralh EJ, Slezak JM, Rohlinger AL, Olson JB, Milliner DS (2005) International registry for primary hyperoxaluria. Am J Nephrol 25:290-296

6. Jamieson NV (2005) A 20-year experience of combined liver/ kidney transplantation for primary hyperoxaluria $(\mathrm{PH} 1)$ : the European PH1 transplant registry experience 1984-2004. Am J Nephrol 25:282-289

7. Jamieson NV, Jamieson KA (2009) Primary hyperoxaluria type 1: gene therapy by liver transplantation. Transplantation 87:12731274

8. Scheinman JI, Najarian JS, Mauer SM (1984) Successful strategies for renal transplantation in primary oxalosis. Kidney Int 25:804-811

9. Smith LH (1974) Symposium on renal lithiasis. Medical evaluation of urolithiasis. Etiologic aspects and diagnostic evaluation. Urol Clin North Am 1:241-260

10. Scheinman J, Payne R, Booth A (2001) Management of ESRD in primary hyperoxaluria in the U.S.A in 2001: results of dialysis, kidney and combined kidney/liver transplantation (abstract). Kidney Int 60:A1251

11. Cibrik DM, Kaplan B, Arndorfer JA, Meier-Kriesche HU (2002) Renal allograft survival in patients with oxalosis. Transplantation 74:707-710 
12. Scheinman JI, Payne R (2002) ESRD in Sickle Cell Nephropathy, survival with and without transplantation (abstract). J Am Soc Nephrol 13:403A

13. Annual Report of the U.S. Organ Procurement and Transplantation Network and the Scientific Registry of Transplant Recipients (2006) Transplant Data 1996-2005. 2006 OPTN/SRTR Annual Report 1996-2005. Health Resources and Services Administration, Healthcare Systems Bureau, Division of Transplantation, Rockville

14. Annual Report of the U.S. Organ Procurement and Transplantation Network and the Scientific Registry of Transplant Recipients (2008) Transplant Data 1996-2007. 2008 OPTN/SRTR Annual Report 1996-2007. Health Resources and Services Administration, Healthcare Systems Bureau, Division of Transplantation, Rockville

15. Adam R, Hoti E (2009) Liver transplantation: the current situation. Semin Liver Dis 29:3-18

16. Milliner DS, Monico CG, Bergstralh E, Herges R, Hoppe B, Langman CB, Lieske JC (2008) Transplantation strategy in primary hyperoxaluria (abstract SA-FC446). J Am Soc Nephrol 19:101A

17. Brinkert F, Ganschow R, Helmke K, Harps E, Fischer L, Nashan B, Hoppe B, Kulke S, Muller-Wiefel DE, Kemper MJ (2009)
Transplantation procedures in children with primary hyperoxaluria type 1: outcome and longitudinal growth. Transplantation $87: 1415-1421$

18. Sutherland SM, Alexander SR, Sarwal MM, Berquist WE, Concepcion W (2008) Combined liver-kidney transplantation in children: indications and outcome. Pediatr Transplant 12:835-846

19. Marangella M (1999) Transplantation strategies in type 1 primary hyperoxaluria: the issue of pyridoxine responsiveness. Nephrol Dial Transplant 14:301-303

20. Scheinman JI (1991) Primary hyperoxaluria: therapeutic strategies for the 90's. Kidney Int 40:389-399

21. Scheinman JI, Payne R, Booth A (2001) Management of ESRD in primary hyperoxaluria in the U.S.A in 2001: results of Dialysis, kidney and combined kidney/liver transplantation. In: ASN/ISN World Congress of Nephrology.. J Am Soc Nephrol 12:243A

22. Scheinman JI, Voziyan P, Belmont J, Chetyrkin S, Kim D, Hudson B (2005) Pyridoxamine lowers oxalate excretion and kidney crystals in experimental hyperoxaluria: a potential therapy for primary hyperoxaluria. Urol Res 33:368-371

23. Hoppe B, Beck B, Gatter N, von Unruh G, Tischer A, Hesse A, Laube N, Kaul P, Sidhu H (2006) Oxalobacter formigenes: a potential tool for the treatment of primary hyperoxaluria type 1 . Kidney Int 70:1305-1311 